\title{
Voice Based E-Mail System
}

\section{Shreyas J, Deepthi P D'souza, Poojary Shashank Chandrahas}

Computer Science Department, Srinivas Institute of Technology, Mangalore, Karnataka, India

\section{ABSTRACT}

Article Info

Volume 7, Issue 4

Page Number: 159-163

Publication Issue :

July-August-2021

\section{Article History}

Accepted : 06 July 2021

Published : 13 July 2021
In today's world, communication has become very easy due to integration of communication technologies with internet. However, the visually challenged people find it very difficult to utilize this technology because of the fact that using it requires visual perception. Even though much advancement has been implemented to help them use computers efficiently, no user who is visually challenged can use this technology as efficiently as a normal user since they require some practice for using the available technologies. This project aims at developing an email system that will help even a naïve, visually impaired person to use the services for communication without previous training. The system does not require the use of keyboard. Instead, it will work only on mouse operations and speech. This system can also be used by any normal person, for instance, by someone who is unable to read.

Keywords : Python, Text to speech, Django

\section{INTRODUCTION}

Internet plays an important role in today's world of communication. Today the planet is running on the idea of internet. No work are often avoided use of internet. electronic message i.e., email is that the most vital part in day-to-day life. But a number of the people in today's world don't skills to form use of internet, some are blind or some are illiterate. So, it goes very difficult to them when to measure during this world of internet. Nowadays there are various technologies available during this world like screen readers, ASR, TTS, STT, etc. but these aren't that much efficient for them. Around 39 million people are blind and 246 people have low vision and also 82 of individuals living with blindness are 50 aged and above. we've to form some internet facilities to them in order that they can use internet. Therefore, we came up with our project as voice-based email system for blinds which can help tons to visually impaired peoples and also illiterate peoples for sending their mails. The users of this technique don't got to remember any basic information about keyboard shortcuts also as location of the keys. Simple click operations are needed for functions making system easy to use for user of any age bracket. Our system provides location of where user is prompting through voice in order that user doesn't need to worry about remembering which click operation, he/she wants to realize . within the previous system with the assistance of screen readers it's difficult for blind man to access E-mail system and computer operating easily because it's noisy audio interface. 
These available systems require use of keyboard which is extremely difficult for blind people to acknowledge and remember characters of keyboard. So, we implement voice-based E-mail system for blind man and it also helps handicapped and illiterate people. This project aims at developing an email system which will help even a naïve, visually impaired person to use the services for communication without previous training. The system doesn't require the utilization of keyboard. Instead, it'll work only on mouse operations and speech conversion to text. this technique also can be employed by any normal person, as an example, by someone who is unable to read.

\section{RELATED WORK}

The earlier work implements creation of programs simulating human communication remains relevant. The simplest model of communication is that the database of questions and answers to them. In this case, there's the matter of describing the knowledge domain and therefore the implementation of the interpreter program. The mark-up language of the knowledge base can include question patterns and corresponding response patterns, as well as the background history of the dialogues to them and the name of the corresponding topic of communication. Another research paper aims to develop a search engine which supports Man-Machine interaction purely in the form of voice. A novel Voice based program and Web-page Reader which allows the users to command and control the online browser through their voice, is introduced. The existing Search Engines get request from the user within the sort of text and respond by retrieving the relevant documents from the server and displays within the sort of text. Even though the prevailing web browsers are capable of playing audios and videos, the user has got to request by typing some text within the search text box then the user can play the interested audio/video with the help of Graphical User Interfaces (GUI).The related old work implements systems working on speech Recognition are highly dependent on training done by the speaker. They only answer the input given by the actual speaker making the system speaker dependent. Different techniques have been used to enhance the system and to make its response more efficient and accurate i.e., Hidden Markov Model (HMM), MelCepstral feature extraction algorithm and clustering techniques. Further adding to the recognition rate has developed a dictation pad for which they conducted an experiment in which they prove that age of the speaker also affects the recognition rate of the system. The proposed system which emphasizes more on user friendliness of normal users, our system focuses more on user friendliness of types of people including normal people visually impaired people as well as illiterate people. This system enables the user to connect the files using Speech Recognition in mail not by the Screen Readers which is merely use to login into the mail. This system are going to be perfectly accessible to all or any sorts of users because it is simply supported simple mouse clicks and speech inputs and there's no got to remember keyboard shortcuts. The process used to build this system is Windows Speech Recognition which allows a user to control a computer, including the operating system desktop user interface, through voice commands. Applications, including most of these are bundled with Windows, can also be controlled through voice commands.

\section{SYSTEM IMPLEMENTATION}

The system is implemented with Django framework and in python programming language. 
The proposed system is designed with web user interface.The user has to provide a voice command input to the proposed system, the system will convert the audio input into textual format for processing.The input is also preprocessed in order to remove input mismatches and grammactical mistakes. Onces user provides the credentials for logging into the system, a secure web socket connection is established to the user mail server . A two way handshake is enabled to perform GET/POST request from the mail servers. On successful login the user is directed to a user option page where the proposed system reads out the user option via its audio output device, in order user to provide future inputs. The user can now provide the voice command for respective user option. On receiving the voice command from user, the input is validated and preprocessing to input is done and its converted from audio to text format.

The user is provided with options like reading the inbox mails, composing a mail, reading of sent mails as well as deletion of mails. On selection on any options GET/POST request is made via by Simple Mail Transfer Protocol . The result of the respective operations selected by the user is rendered in form of audio output via audio output device.

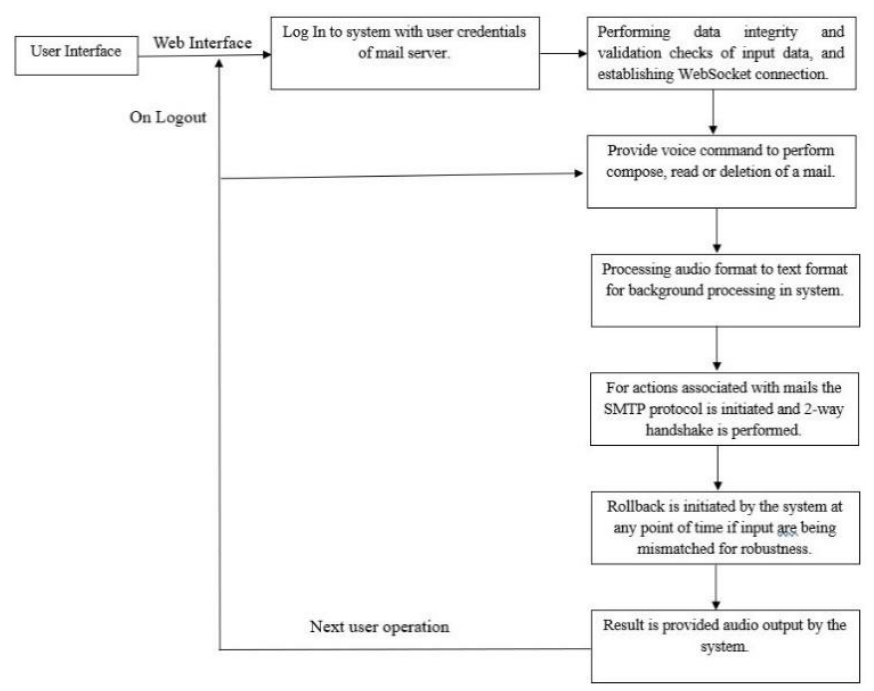

Figure 1. System architecture of proposed system.
The proposed system architecture is designed to be robust in scenario of varying input mismatches or in case of internal server error of mail server or in case of disconnections. The system exist from mail server on logout voice command by user, after which the web socket is disconnected and SMTP protocol revokes the handshake between application and mail server.

\section{EXPERIMENTAL RESULTS}

Proposed system is developed and following experimental result is obtained.

Figure 2 shows the home page of proposed system UI, here system renders voice output providing guidelines regarding login for system i.e email id and password of user mail server.

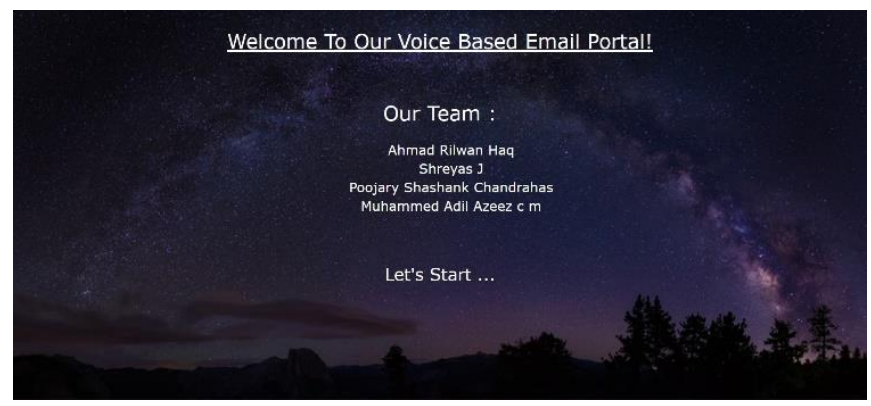

Figure 2. Homepage of proposed system.

Figure 3 describes the user options available once after user is logged in, in the phase the proposed system provides voice instruction for the user to select the required user options.

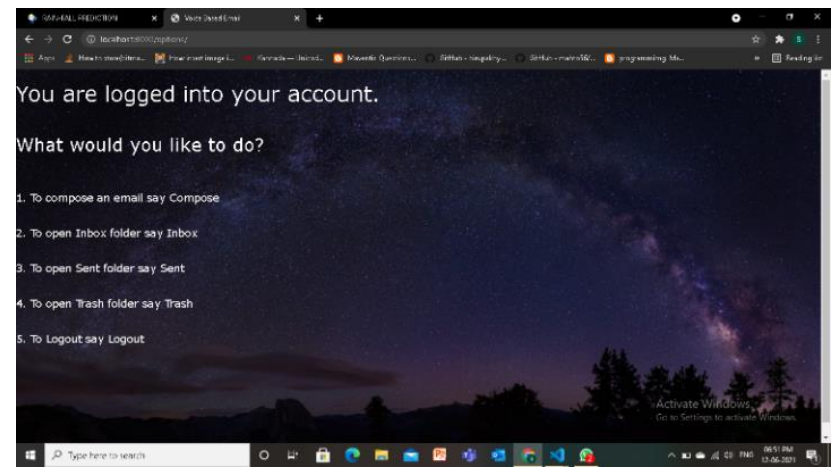

Figure 3. User option page of proposed system 
Figure 4 describes the user option Composing of email, in the phase the proposed system takes user input via voice command for details like receiver email id, attachment details, messages etc.

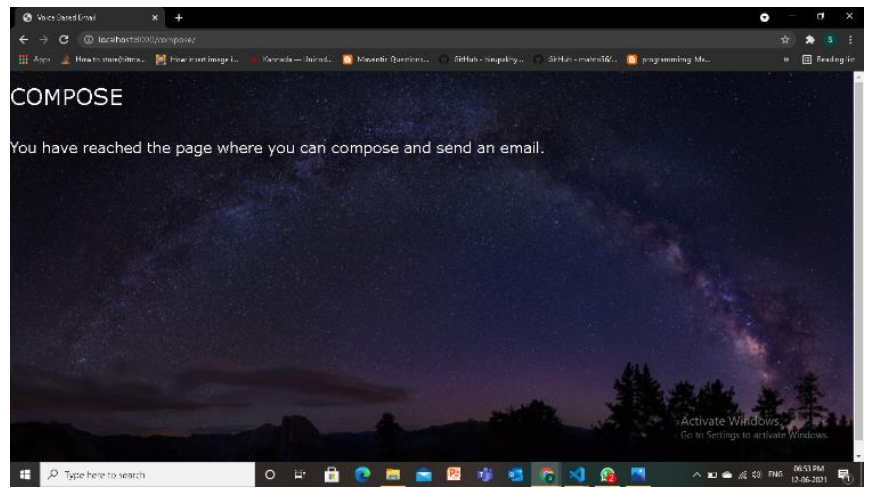

Figure 4. Compose page of proposed system.

Figure 5 shows the user option reading inbox mails of user, here proposed system provides options like reading of unread mails or search a specific mail or navigate to user option page.

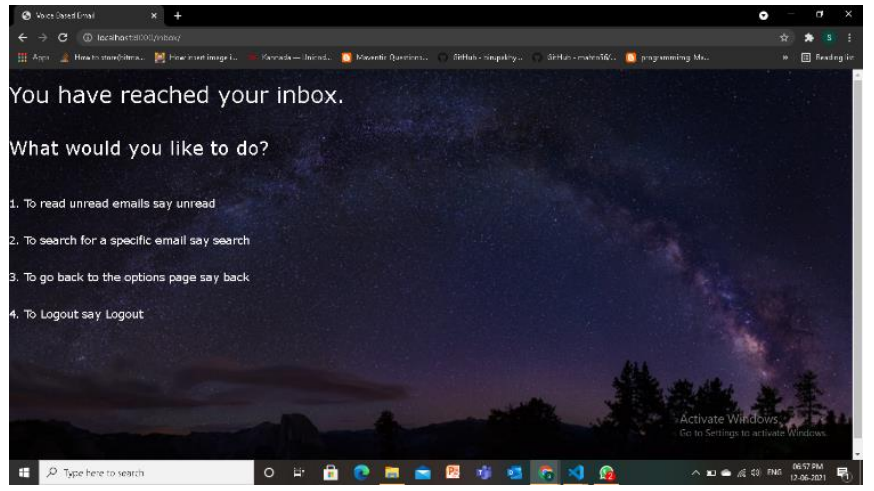

Figure 5. Inbox page of proposed system.

Figure 6 shows the sent mail page were proposed system renders out the mails sent by users.

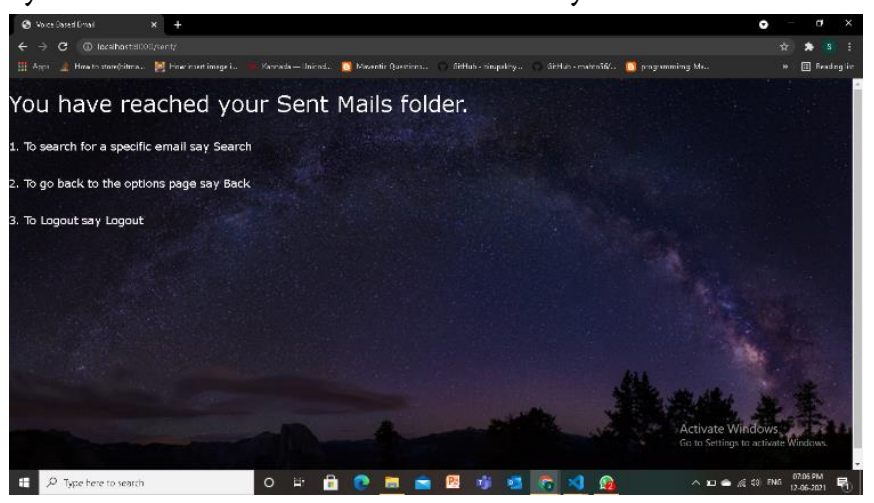

Figure 6. Sent Mail Page of proposed system.
Figure 7 shows the trash page of proposed system were the system reads out the mails that are present in the trash bin of user mail server.

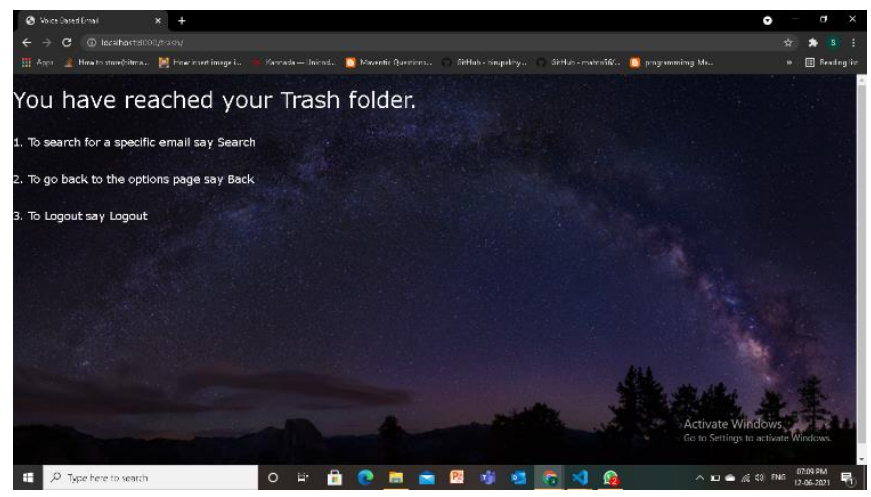

Figure 7. Trash Mail Page of proposed system.

\section{CONCLUSION}

The problem of physically disabled candidates is being bridged by creating an web application. This web app allows these users to interact with real world with the voice commands. It allows the user to perform mail transaction like another normal user being used in mail server. It bridges the gap between the mutual communication issues caused due to lack of vision impaired by the user. This web app results in efficient usage of time. The system helps to improve the performance. Maintaining the project is easy and manageable. It is easily understandable by the user.Future work that can be added to this project may be, the web app can be converted into android applications. It can be modified in such a way that application can be used by organizations other than educational institutions. Organizations can add functionalities depending on their needs.

\section{REFERENCES}

[1]. Voice Based Search Engine And Web Page Reader. 1,Ummuhanysifa U 2, Nizar Banu P K 1,2,B.S. Abdur Rahman University Chennai. 
[2]. Development of the Speech-to-Text Chatbot Interface Based on Google API Nataliya Shakhovska , Oleh Basystiuk , Khrystyna Shakhovska Lviv Polytechnic National University, Lviv 79013, Ukraine.

[3]. VoiceMail Architecture in Desktop and Mobile Devices for the Blind People Tirthankar Dasgupta, Aakash Anuj, Manjira Sinha, Ritwika Ghose, Anupam Basu Indian Institute of Technology Kharagpur.

[4]. Voice Based Interactive System for Visually Impaired Sadaf Abdul Rauf, Mahnoor Yaqoob, Ayesha Qurban Dept. of Software Engineering Fatima Jinnah Women University Rawalpindi, Pakistan.

[5]. Voice based mail attachment for visually challenged people 1 Tharani K K, 2 Shalini R, 3 Jeyanthi I, 4 Dr.Deepalakshmi R, 1,2,3,4 Department of Computer Science and Engineering, Velammal College of Engineering and Technology, Madurai, Tamil Nadu, India.

[6]. Audio-Enabled Graphical User Interface for The Blind or Visually Impaired Frank McKiel Jr,2010.

\section{Cite this article as :}

Shreyas J, Deepthi P D'souza, Poojary Shashank Chandrahas, "Voice Based E-Mail System", International Journal of Scientific Research in Computer Science, Engineering and Information Technology (IJSRCSEIT), ISSN : 2456-3307, Volume 7 Issue 4, pp. 159-163, July-August 2021. Available at doi : https://doi.org/10.32628/CSEIT217441

Journal URL : https://ijsrcseit.com/CSEIT217441 